\section{Do RET somatic mutations play a role in Hirschsprung disease?}

To the Editor: We read "RET Somatic Mutations Are Underrecognized in Hirschsprung Disease," by Jiang et al. ${ }^{1}$, recently published in your journal, with great interest. The authors hypothesize a contribution of RET somatic mutations to Hirschsprung disease (HSCR).

HSCR is a complex inherited disorder characterized by the absence of enteric ganglia in the distal part of the colon. Several genes and loci have been described as underlying disease pathogenesis. However, variants in these genes explain no more than $20 \%$ of all cases. ${ }^{2}$ This missing heritability seen in HSCR is a common feature of many complex disorders, and explaining it remains challenging. Considering that HSCR develops during embryogenesis as a result of either impaired migration, proliferation, or differentiation of enteric neural crest cells (ENCCs), it is tempting to consider that somatic mutations occurring during the development of the enteric nervous system, and specifically affecting ENCCs, can also contribute to HSCR genetics. Jiang and colleagues think that this is possible, and we share that opinion, as we believe that somatic variants could be underrecognized in HSCR, and thus, possibly account for some of the missing heritability. However, the results described by the authors do not, in our opinion, fully support the conclusions of the manuscript. This is mainly because routine genetic testing on DNA derived from blood or saliva would not find these ENCC-specific mutations, nor would it easily detect low mosaic variants. With this letter, we intend to further discuss our concerns and highlight the difference between causative somatic mutations and germ-line mosaicism resulting in seemingly de novo mutations in the next of kin.

In their study, the authors screened 152 HSCR patients using targeted exome sequencing and direct gene screening. In eight patients they identified putative deleterious de novo variants in RET. Because RET is the major HSCR-causing gene, ${ }^{3}$ they assumed that these variants were responsible for the disease phenotype. However, only six patients carried a truly de novo RET mutation; in the other two, the variants identified were also found in one of the parents. The authors continued the genetic analysis of the six remaining patients and determined, based on their findings, that the RET mutations identified were somatic. This result led to the conclusion that RET somatic mosaicism is present in $75 \%$ of these HSCR cases and is, therefore, underrecognized. However, in four of the six patients studied, germ-line mosaicism was identified in the parents. As these variants are transmitted to the affected next of kin as heterozygous variants, they cannot represent somatic mosaicism. In addition, the parents are not affected, leading us to speculate that either their ENCCs are not affected by the mutation or that the mutational load in their ENCCs does not cross a threshold for abnormal ENS development. Therefore, although these four families are likely to represent germ-line mosaicism resulting in seemingly de novo mutations in the next of kin, they do not support the conclusion that RET somatic mutations are underrecognized. These heterozygous mutations would be detected (and are detected in these patients) in routine genetic screening and as such do not resolve missing heritability due to "recognizing" somatic mosaicism.

For the remaining two patients, the RET variants identified were present in blood, saliva, and colon, in family 1 (39/39/ $44 \%)$ and family $2(44 / 35 / 39 \%)$. The authors validated the sequencing data with TA cloning, but the results are variable even within the same tissue, and the differences seen in the amplicon-based sequencing test are well within the normal range for detection of a heterozygous variant. Moreover, the samples tested-blood, saliva, and colon-derive from tissues originating from different germ layers. Blood, mostly lymphocytes, is derived from mesoderm; saliva, lymphocytes, and epithelial lining of the mouth are derived from mesoderm and ectoderm; and the colon has contributions from all three germ layers. The high allele frequencies identified in all three samples for both patients $(>30 \%)$ does not allow for discrimination between very early developmental stage somatic mutations or de novo variants present as a germline mosaicism in one of the parents. Although the authors acknowledge this fact in the discussion, it is more fitting to conclude that the variants identified in these two remaining patients are likely to be de novo heterozygous variants.

In conclusion, we agree with the hypothesis of Jiang et al. and think that somatic variants might well play a role in HSCR development. However, based on the results presented we think that it is not possible to make such a conclusion, as no true somatic RET mutations were identified in any of the presented patients. We believe that to draw the conclusions stated, the allele frequency of the mutations in ENCCs, surface ectoderm, and, for instance, blood or fibroblasts would need to be compared because these represent different germ layers and include the cells involved in HSCR. An experimental setup that would isolate cells specifically from each germ layer, as well as ENCCs, or that would separate the colon into various cell types, would be necessary to determine whether true somatic mutations impact HSCR development. Only if differences were found under such conditions could the authors show that RET somatic mutations are indeed underrecognized in HSCR.

\section{DISCLOSURE}

The authors declare no conflict of interest. 
Erwin Brosens, $P h D^{1,2}$, Katherine C. MacKenzie, MSc ${ }^{1,2}$, Maria M. Alves, $P h D^{1}$ and Robert M.W. Hofstra, $P h D^{1}$

${ }^{1}$ Department of Clinical Genetics, Erasmus University Medical Center, Rotterdam, The Netherlands. Correspondence: Robert M.W. Hofstra (r.hofstra@erasmusmc.nl)

${ }^{2}$ The first two authors contributed equally to this work.

\section{REFERENCES}

1. Jiang $\mathrm{Q}$, Liu F, Miao $\mathrm{C}$, et al. RET somatic mutations are underrecognized in Hirschsprung disease. Genet Med 2018;20:XXX-XXX.
2. Alves MM, Sribudiani Y, Brouwer RW, et al. Contribution of rare and common variants determine complex diseases-Hirschsprung disease as a model. Dev Biol 2013;382:320-9.

3. Amiel J, Sproat-Emison E, Garcia-Barcelo M, et al. Hirschsprung disease, associated syndromes and genetics: a review. J Med Genet 2008;45: $1-14$.

Advance online publication 1 March 2018 2018. doi:10.1038/gim.2018.6 